\title{
Evaluación de un cluster bajo agricultura protegida en México
}

Luz Evelia Padilla-Bernal

Unidad Académica de

Contaduría y Administración,

Universidad Autónoma de

Zacatecas

luze@uaz.edu.mx

Eliver Reyes-Rivas

Unidad Académica de

Contaduría y Administración,

Universidad Autónoma de

Zacatecas

elireyes1508@mns.com

\section{Óscar Pérez-Veyna}

Unidad Académica de Estudios del Desarrollo, Universidad Autónoma de Zacatecas pveyna@uaz.edu.mx

\section{Resumen}

El naciente cluster de tomate bajo agricultura protegida en Zacatecas enfrenta el reto de participar en los mercados globales de una manera sostenible; una respuesta viable para ello es elevar su capacidad de upgrading. En este trabajo se determina la relación entre la eficiencia colectiva en el upgrading del cluster. La información se obtuvo a través de un cuestionario aplicado a técnicos de las unidades de producción (UP), el cual se complementó con entrevistas a propietarios o gerentes, investigadores y funcionarios gubernamentales; posteriormente, se hizo una clasificación de las UP por nivel tecnológico aplicando la técnica de análisis de conglomerado con lo que se obtuvo el índice de eficiencia colectiva, el tipo de gobernanza de las cadenas de valor con las que se relacionan las agroempresas y los tipos de upgrading logrados. Se encontró que las economías externas fueron más dominantes que las acciones conjuntas; la eficiencia colectiva y el upgrading se encuentran positivamente relacionados; asimismo, se observó que el alto nivel tecnológico es condición necesaria, más no suficiente, para que las agroempresas permanezcan competitivas en el mercado global, por lo que se requiere elevar su capacidad de upgrading.

Palabras clave: economías externas, acción conjunta, invernaderos. 


\title{
Efficiency index on protected agriculture cluster in Mexico
}

\begin{abstract}
Protected agriculture producers in Zacatecas are facing the challenge of participating in the global markets with sustainable income. A viable way would be to increase their upgrading capability. This work determines the relationship between upgrading and collective efficiency of the tomato cluster under protected agriculture in Zacatecas. Information was obtained through a survey among technicians, complemented with interviews |with owners of the production units, researchers and government authorities. Applying cluster analysis technique, a classification of production units by technological level was made. By these means we obtained the collective efficiency index, the value chain pattern of governance of the value chain in which enterprises participate and the upgrading pattern. External economies were more dominant than joint actions. Collective efficiency and upgrading were positively correlated. A high technological level is a necessary but insufficient condition for enterprises competitiveness in the global market, so that an increase in upgrading capability is required.
\end{abstract}

Keywords: external economies, joint action, greenhouses.

\section{Antecedentes}

La globalización y la apertura comercial, además de crisis alimentaria y financiera, han cambiado de forma radical el ámbito económico y empresarial. En el caso del sector agroalimentario, existen otros factores que también lo están impactando: la modificación en la asignación de apoyos gubernamentales, los grandes avances tecnológicos (informática, microelectrónica, biotecnología, ingeniería genética, nanotecnología y telecomunicaciones), una mayor preocupación por el cuidado del medio ambiente y una demanda por productos más diferenciados (Brambila, 2006; Gomes, 2006), regida por criterios de calidad, inocuidad, conveniencia y nutrición (Kinsey, 2005; Suárez y Bejarano, 2001). Esta situación no es ajena a los cultivos producidos bajo agricultura protegida y con alta demanda en los mercados internacionales, como lo es el tomate de invernadero (Kaufman et al., 2000; Calvin y Cook, 2001; Cook y Calvin, 2005).

La agricultura protegida, que implica una amplia gama de métodos de producción ejerciendo algún grado de control sobre varios factores del medio ambiente, ha cobrado gran importancia en los últimos años en el estado de Zacatecas; esta alter- 
nativa se ha empezado a considerar por las autoridades gubernamentales como una posibilidad para coadyuvar en el desarrollo del estado. En Zacatecas, al igual que en otras regiones de México, los sistemas de producción bajo agricultura protegida han crecido aceleradamente en los últimos años. La tasa media de crecimiento anual (TMCA) de la superficie cultivada, durante el periodo de 2001 a 2007, fue del 30.5\%. Actualmente, se estima que esta superficie asciende a más de 200 hectáreas, de ellas en 2007 el 95\% se cultivó con tomate (Padilla-Bernal, RumayorRodríguez, Pérez-Veyna y Reyes-Rivas, 2008), encontrándose más del 95\% en la región del altiplano zacatecano.

Algunas de las principales causas de la rápida expansión de la superficie de tomate bajo agricultura son las siguientes: el rendimiento potencial sobre la inversión que estos sistemas de producción pudieran ofrecer por la ubicación de sus regiones productoras; las condiciones agroclimáticas que permiten alargar el ciclo sin incurrir en altos costos de combustible; la cercanía a la frontera de los EE.UU. principal mercado de exportación para los tomates mexicanos; y las facilidades otorgadas por los diferentes niveles de gobierno para su construcción. El gobierno estatal en sus dos últimos planes estatales de desarrollo (1999-2004 y 2005-2010) ha planteado como parte de las estrategias para la reactivación del campo la promoción de la agricultura protegida. Los apoyos gubernamentales para la construcción de unidades de producción iniciaron en 2000 y han mostrado un comportamiento creciente en el tiempo.

Con base en las características del crecimiento de las unidades de producción bajo agricultura protegida en Zacatecas y el dinamismo económico que se pretende generar a su alrededor, se puede decir que se está presentando la primera fase del desarrollo del cluster de tomate. Los clusters son empresas próximas geográficamente involucradas en relaciones verticales y horizontales, lo que implica simultáneamente que las instituciones de soporte comparten una misma visión de desarrollo de las actividades, basada en competencia y cooperación en un mercado específico (Cooke y Huggins, 2002); sin embargo, Visser (2004) señala que sólo en los clusters más desarrollados, los actores interactúan con propósitos estratégicos, cooperan y aprenden a hacerlo, institucionalizando sus esfuerzos colaborativos.

La existencia de aglomeración de pequeñas y medianas empresas concentradas geográficamente y especializadas sectorialmente es una situación común en los países en desarrollo, aunque esto no implica que esos clusters compartan las ventajas de los distritos industriales exitosos. El cluster puede ser considerado como 
un factor facilitador de una serie de subsecuentes hechos, los cuales pueden o no suceder: división y especialización de trabajo, la emergencia de una amplia red de proveedores, la aparición de agentes quienes venden a mercados nacionales e internacionales, la emergencia de proveedores de servicios especializados, la materialización de un grupo de trabajadores especializados y la formación de asociaciones de negocios (Giuliani, Pietrobelli y Rabellotti, 2005). Con el fin de capturar los impactos positivos de esas características en la competitividad de las empresas que forman un cluster, Schmitz (1995) desarrolló el concepto de eficiencia colectiva, entendido como la ventaja competitiva derivada de las economías externas locales y la acción conjunta (Pietrobelli y Rabellotti, 2006). La integración del cluster del tomate bajo agricultura protegida ofrece oportunidades para que las agroempresas que lo integran se apropien de las economías externas y se facilite el desarrollo de acciones conjuntas entre los actores locales.

Los cambios generados en los mercados financieros y canales de distribución, así como en las tecnologías de información sugieren mayor atención en los nexos externos. A través de la cadena global de valor se pueden tomar en cuenta las actividades que se llevan a cabo fuera del cluster y entender el papel estratégico de las relaciones con actores externos claves. Considerando las relaciones entre los diversos actores involucrados en la cadena, el concepto de gobernanza es central para el análisis. Desde este esquema, el gran reto al que se enfrentan las unidades de producción bajo agricultura protegida es cómo participar en los mercados globales de tal forma que mantengan un crecimiento del ingreso sostenible; es decir, mantenerse competitivas. Diversos autores sobre el tema de competitividad (Pietrobelli y Rabellotti, 2006) sugieren que la respuesta más viable es hacer mejores productos de forma más eficiente, o hacer actividades más especializadas; es decir, elevar su capacidad de upgrading, la cual se define como innovar para incrementar valor agregado (Giuliani et al., 2005; Humphrey y Schmitz, 2002; Pietrobelli y Rabellotti, 2006).

Es así que el objetivo de este trabajo es determinar la relación entre la eficiencia colectiva y el upgrading del cluster de tomate bajo agricultura protegida de Zacatecas, así como identificar los patrones de gobernanza de la cadena de valor en la que participan. Esto con el fin de proveer información que coadyuve a la propuesta de políticas públicas para apoyar el upgrading de las unidades de producción que les permita permanecer competitivas en el mercado global, lo que impactaría en el crecimiento económico del estado. 
Se partió de las siguientes preguntas de investigación: ¿son las unidades de producción bajo agricultura protegida con mayor nivel tecnológico las que presentan mayor interrelación entre la eficiencia colectiva y el upgrading? y ¿qué acciones pudieran emprenderse para apoyar el upgrading de las unidades de producción bajo agricultura protegida?

\section{La eficiencia colectiva del cluster y el upgrading}

La eficiencia colectiva (EC) se desarrolla a través de las incidentales economías externas (EEs) y las conscientemente perseguidas acciones conjuntas (AC). El concepto de EEs fue introducido por primera vez por Alfred Marshall para explicar por qué y cómo la elección de la localización de la industria es un factor importante, y por qué y cómo las pequeñas y medianas empresas pueden ser eficientes y competitivas (Schmitz, 1995). Las economías externas se refieren al impacto positivo o negativo que una empresa puede tener en otra de manera involuntaria o pasiva; éstas son esenciales para entender las ventajas que tienen las empresas al encontrase en un cluster. De acuerdo con Pietrobelli, Rabellotti y Giuliani (2006) en los clusters las economías externas más comunes son: a) la creación de un mercado de mano de obra especializada; b) la creación de un mercado de insumos, maquinaria e insumos especializados; c) acceso a un mercado por mejores productos; y d) un acceso más fácil al conocimiento especializado en tecnologías y mercados, así como un rápido flujo de la información.

Por otra parte, las empresas ubicadas en los clusters también realizan acciones conjuntas (AC) y se caracterizan por desarrollarse en forma planeada y consciente. En este tipo de acciones se requieren inversiones específicas y compromiso de las empresas, pues en la medida que se enfrentan a nuevos competidores adoptan innovaciones o ingresan a nuevos mercados. De acuerdo con Navdi (1999), las acciones conjuntas pueden ser de tres tipos: a) la acción conjunta dentro del encadenamiento bilateral horizontal entre dos o más productores locales que puede incluir comercialización conjunta de productos, compra conjunta de insumos, órdenes compartidas, uso común de equipo especializado, desarrollo de nuevos productos e intercambio de experiencia e información de mercados; b) acción conjunta dentro del encadenamiento vertical, donde la cooperación se da hacia atrás de la cadena con productores y hacia adelante, con comercializadores y compradores; c) la acción conjunta dentro del encadenamiento multilateral horizontal, que se presenta dentro de un gran número de productores locales; esta última considera 
la cooperación entre grupos o asociaciones de negocios y centros de servicio de desarrollo de negocios.

La combinación de las economías externas incidentales y los efectos de la cooperación activa determinan el grado de la eficiencia colectiva de un cluster, lo que conlleva el potencial para el upgrading de las empresas (Giuliani et al., 2005). El concepto de upgrading se refiere al hacer mejores productos, hacerlos más eficientemente, trabajar en actividades más especializadas, o generar bienes con mayor nivel tecnológico. Dicho concepto se empezó a emplear en el marco de los estudios de competitividad internacional y su uso se ha extendido también a los niveles empresa y región (Coelho, 2007). El upgrading está estrechamente relacionado a la innovación de productos, de procesos y funcional, que permite mejorar el valor agregado. Humphrey y Schmitz (2000) distinguen cuatro tipos de upgrading: 1) de procesos, que se refiere a la transformación más eficiente de insumos en productos o la producción de tecnología superior; 2) de productos, que consiste en desarrollar líneas de producción más sofisticadas en términos de aumentar su valor agregado; 3) funcional, que se refiere a la adquisición de nuevas funciones dentro de la cadena de valor; y 4) intersectorial, que consiste en aplicar la competencia adquirida en una función particular en un nuevo sector.

Pertenecer a un cluster o participar en una cadena de valor puede facilitar el aprendizaje y el upgrading sostenible; sin embargo, el papel de cada uno de esos factores puede cambiar de acuerdo con las especificidades del sector industrial del cluster y, en especial, con base en sus características tecnológicas; el upgrading puede diferir dependiendo de las características del sector industrial. Pietrobelli y Rabellotti (2004, 2006), siguiendo la taxonomía de Pavitt, identificaron cuatro sectores industriales para América Latina: a) manufactura tradicional, b) basado en recursos naturales, c) industrias de productos complejos y d) proveedores especializados. La industria del tomate bajo agricultura protegida puede ser ubicada dentro del sector basado en recursos naturales.

Aunque el enfoque del presente trabajo se refiere al análisis de las relaciones verticales y horizontales intracluster que generan eficiencia colectiva en el cluster del tomate bajo agricultura protegida, los recientes cambios en los sistemas de producción, canales de distribución y los mercados financieros, acelerados por la globalización de los mercados de productos y la brecha en las tecnologías de información sugieren poner atención en las relaciones externas (Giuliani et al., 2005) 
debido a que las empresas y clusters están cada vez más integradas en cadenas de valor que operan en diferentes países que tienen alcance global. Para este fin la cadena de valor (CV) ayuda a tomar en cuenta las actividades externas al cluster y en particular entender el papel de las relaciones con agentes externos clave (Pietrobelli y Rabellotti, 2006). Para las empresas pequeñas, la participación en cadenas de valor es una forma de obtener la información requerida para ganar acceso en el mercado global.

El enfoque de la cadena de valor se requiere para el análisis de las relaciones entre los actores involucrados en la cadena y sus implicaciones para el desarrollo. En el estudio de dichas relaciones, el concepto de gobernanza o coordinación es fundamental para el trabajo. De igual forma, el análisis de la participación e involucramiento proactivo de todos los actores de la cadena de valor es crucial. En cualquier punto de la cadena, algún grado de gobernanza es requerido para decidir sobre qué se va a producir, cómo se va a producir, y cuánto se va producir (Pietrobelli y Rabellotti, 2006). Ahora bien, la gobernanza puede ocurrir a través de relaciones de mercado o de relaciones ajenas al mercado. De acuerdo con Humphrey y Schmitz (2000) en las relaciones ajenas al mercado se pueden presentar tres tipos de gobernanza: a) relaciones de redes, lo que implica cooperación entre firmas con niveles de poder más o menos semejantes y comparten sus competencias dentro de la cadena; b) relaciones de cuasi-jerarquía, las que ocurren entre empresas legalmente independientes en donde una es subordinada a otra (usualmente el comprador) con un líder en la cadena definiendo reglas en productos y procesos que el resto de los actores debe seguir; y c) relaciones de jerarquía que se presenta cuando una empresa toma propiedad de otra o establece su propia compañía dentro del cluster. Humphrey y Schmitz (2000) consideran que tanto el cluster como la cadena de valor ofrecen oportunidades para el upgrading y la modernización de las pequeñas y medianas empresas locales. Aunque debe señalarse que con el fin de evaluar la contribución potencial de los clusters y las cadenas de valor a la innovación y upgrading de estas empresas, se requiere entender la organización del encadenamiento de las empresas y la estructura de la gobernanza de las cadenas de valor, así como el conocimiento de las características del sector industrial al que pertenecen.

\section{Materiales y métodos}

Con el fin de determinar la forma en que el upgrading de las empresas de agricultura protegida en Zacatecas es afectado por la eficiencia colectiva del cluster en el 
que operan e identifican los patrones de gobernanza de la cadena de valor en la que participan se empleó la metodología propuesta por Giuliani et al. (2005) y Pietrobelli y Rabellotti (2006), quienes analizaron el grado de eficiencia colectiva y los niveles de upgrading en los clusters latinoamericanos.

La información requerida se obtuvo a través de un cuestionario que se aplicó a 45 técnicos de las unidades de producción bajo agricultura protegida durante el periodo de marzo a mayo de 2008 y se complementó con diez entrevistas con los propietarios o gerentes. Además, durante los meses de mayo a agosto del mismo año se realizaron entrevistas a dos investigadores del INIFAP y uno de la Universidad Autónoma de Zacatecas (UAZ), así como a cinco funcionarios relacionados con programas de agricultura protegida de instituciones gubernamentales en el Estado. La información recabada en el trabajo de campo fue complementada con fuentes secundarias tales como publicaciones y reportes. Los criterios para seleccionar las unidades de producción sujetas a estudio fueron: a) contar con una superficie mayor o igual a 2,500 $\mathrm{m}^{2}$; b) producir hortalizas, se excluye la producción de plántula y flores; y c) disponibilidad de los involucrados para contestar las preguntas. El marco de análisis fue el Altiplano Zacatecano en donde en 2007 se cultivaron 174.1 ha con tomate bajo agricultura protegida, distribuidas en 45 unidades de producción.

Los entrevistados se autoevaluaron contestando a grupos de preguntas relacionadas con el objeto de estudio: eficiencia colectiva, tipo de gobernanza de las cadenas de valor con las que se relacionan las agroempresas y tipos de upgrading logrados. Para cuantificar el grado de eficiencia colectiva fueron evaluadas las economías externas y las acciones conjuntas, obteniéndose los índices correspondientes. Se aplicó una escala tipo Likert de acuerdo con lo siguiente: totalmente de acuerdo=3, parcial acuerdo $=2$, desacuerdo $=1$, y no hay o no existe $=0$. Los indicadores de economías externas fueron: mercado de trabajo especializado, disponibilidad local de insumos, facilidad de acceso a información y de acceso a mercados. En el caso de la acción conjunta los indicadores fueron: relación vertical hacia atrás, relación vertical hacia adelante, relación horizontal bilateral y relación horizontal multilateral. Los índices de economías externas y acción conjunta fueron obtenidos a través de la suma de los valores de cada uno de los indicadores. El índice de eficiencia colectiva (IEC) fue el promedio simple de los índices de economías externas (IEE) y acción conjunta (IAC). Para la evaluación del upgrading se hizo uso de la escala ya mencionada; además, se hicieron bloques de preguntas referentes a los cuatro tipos de upgrading: producto, proceso, funcional e intersectorial. Finalmente, se 
identificó el tipo de gobernanza de la cadena de valor — mercado, red, cuasi-jerarquía y jerarquía - en las que las agroempresas participan. La información obtenida en las entrevistas a los técnicos fue complementada y cruzada con las entrevistas a los investigadores y funcionarios públicos. Para determinar la relación entre eficiencia colectiva y upgrading se obtuvo el índice de correlación Rho de Spearman de la estadística no paramétrica. La aplicación de los métodos no paramétricos obedece al tamaño de la muestra y a la ausencia de normalidad en los datos, lo cual condiciona el uso de las pruebas paramétricas.

El índice de eficiencia colectiva y el nivel de upgrading también se obtuvieron agrupando a las agroempresas por nivel tecnológico. Para la agrupación de las empresas por nivel tecnológico se aplicó la técnica análisis de conglomerados. Los conglomerados se determinaron a través del procedimiento de análisis jerárquico con el método de enlace entre grupos, haciendo uso del programa estadístico SPSS v16. Las variables consideradas para la obtención de los conglomerados fueron: estructura, control de clima, forma de cultivo y tamaño ${ }^{1}$.

\section{Resultados}

Con base en el informe de aglomeración y la gráfica dendrograma se definieron cuatro grupos de las unidades de producción: baja tecnología, tecnología intermedia, transición tecnológica y alta tecnología (cuadro 1).

\section{Eficiencia colectiva}

El cluster del tomate bajo agricultura protegida en Zacatecas, al igual que las otras industrias basadas en recursos naturales de América Latina, tiene algunas características de las industrias basadas en ciencia, las cuales cambian e innovan con base en descubrimientos científicos y su consecuente upgrading tecnológico. En esta industria, el grueso de la investigación es desarrollada por laboratorios de investigación en biotecnología y químicos, así como en centros de investigación y posteriormente se hace llegar a las unidades de producción (Pietrobelli et al., 2006).

\footnotetext{
${ }^{1}$ Las unidades de producción se clasificaron por tamaño siguiendo el criterio de la Comisión Técnica del Programa de Invernaderos SEDAGRO-SAGARPA en Zacatecas: a) pequeño, con una superficie hasta de $2500 \mathrm{~m}^{2}$; b) mediano, mayor de $2500 \mathrm{~m}^{2}$ hasta $1.5 \mathrm{ha}$, y c) grande, mayor de 1.5 ha.
} 


\section{Cuadro 1}

Unidades de producción con agricultura protegida en Zacatecas agrupadas por nivel tecnológico ${ }^{1}$ considerando las variables de obtención del conglomerado

\begin{tabular}{|c|c|c|c|c|c|c|}
\hline Variable & Tipo & $\begin{array}{c}\text { Baja } \\
\text { tecnología }\end{array}$ & $\begin{array}{l}\text { Tecnología } \\
\text { intermedia }\end{array}$ & $\begin{array}{l}\text { Transición } \\
\text { tecnológica }\end{array}$ & $\begin{array}{c}\text { Alta } \\
\text { tecnología }\end{array}$ & Total \\
\hline \multicolumn{7}{|l|}{ Estructura } \\
\hline & $\begin{array}{l}\text { Raspa y } \\
\text { amagado }\end{array}$ & 23 & & & & 23 \\
\hline & Multitúnel & & 12 & 3 & 6 & 21 \\
\hline & Total & 23 & 12 & 3 & 6 & 44 \\
\hline \multicolumn{7}{|l|}{ Control de clima } \\
\hline & Automatizado & & 2 & 1 & 5 & 8 \\
\hline & Mecánico & 2 & 5 & & 1 & 8 \\
\hline & Manual & 21 & 5 & 2 & & 28 \\
\hline & Total & 23 & 12 & 3 & 6 & 44 \\
\hline \multicolumn{7}{|l|}{ Forma de cultivo } \\
\hline & Hidroponía & & & & 6 & 6 \\
\hline & Suelo & 23 & 12 & & & 35 \\
\hline & $\begin{array}{l}\text { Suelo e } \\
\text { hidroponía }\end{array}$ & & & 3 & & 3 \\
\hline & Total & 23 & 12 & 3 & 6 & 44 \\
\hline \multicolumn{7}{|l|}{ Tamaño } \\
\hline & Pequeño & 5 & 1 & & & 6 \\
\hline & Mediano & 7 & 8 & 1 & 1 & 17 \\
\hline & Grande & 11 & 3 & 2 & 5 & 21 \\
\hline & Total & 23 & 12 & 3 & 6 & 44 \\
\hline
\end{tabular}

Nota: ${ }^{1 /}$ No se incluye la unidad de producción con estructura de macrotúnel.

Fuente: Elaboración propia con base en datos obtenidos en el trabajo de campo.

En el cuadro 2 se presenta el IEC de las unidades de producción agrupadas por nivel tecnológico, obtenido como una combinación del IEE y el IAC. De acuerdo con lo que la teoría predice, se observa que en todos los grupos las economías externas son más dominantes que las acciones conjuntas, situación similar a lo encontrado por Giuliani et al. (2005). El IEC se encuentra 50\% debajo del valor máximo esperado (12), sólo en el grupo de alta tecnología este índice alcanza un valor superior. En este grupo la principal fuente de economías externas es el mercado de trabajo local y el acceso al mercado. En cambio, en los tres grupos tecnológicos restantes, la disponibilidad de insumos locales es lo que más contribuye a la generación de las economías externas. Las unidades de producción con menor nivel tecnológico son beneficiadas en menor medida por el acceso a la información. 


\section{Cuadro 2}

\section{Índice de eficiencia colectiva de las unidades de producción agrupadas por nivel tecnológico}

\begin{tabular}{|c|c|c|c|c|c|}
\hline Concepto & $\begin{array}{c}\text { Baja } \\
\text { tecnología }\end{array}$ & $\begin{array}{l}\text { Tecnología } \\
\text { intermedia }\end{array}$ & $\begin{array}{l}\text { Transición } \\
\text { tecnológica }\end{array}$ & $\begin{array}{c}\text { Alta } \\
\text { tecnología }\end{array}$ & $\begin{array}{c}\text { Promedio } \\
\text { total }\end{array}$ \\
\hline \multicolumn{6}{|l|}{ Economías externas } \\
\hline $\begin{array}{l}\text { Mercado de trabajo } \\
\text { especializado }\end{array}$ & 1.6 & 1.6 & 1.9 & 2.3 & 1.7 \\
\hline $\begin{array}{l}\text { Disponibilidad de } \\
\text { insumos }\end{array}$ & 1.9 & 1.9 & 1.9 & 1.8 & 1.9 \\
\hline Acceso a información & 1.4 & 1.6 & 1.3 & 1.9 & 1.5 \\
\hline Acceso al mercado & 1.3 & 1.0 & 0.9 & 2.0 & 1.3 \\
\hline $\begin{array}{l}\text { Índice de economías } \\
\text { externas }\end{array}$ & 6.2 & 6.1 & 6.0 & 8.0 & 6.4 \\
\hline \multicolumn{6}{|l|}{ Acción conjunta } \\
\hline $\begin{array}{l}\text { Relación vertical hacia } \\
\text { atrás }\end{array}$ & 1.9 & 1.7 & 1.0 & 1.8 & 1.8 \\
\hline $\begin{array}{l}\text { Relación vertical hacia } \\
\text { adelante }\end{array}$ & 2.0 & 2.0 & 2.0 & 2.2 & 2.0 \\
\hline $\begin{array}{l}\text { Relación horizontal } \\
\text { bilateral }\end{array}$ & 0.6 & 0.8 & 0.3 & 0.9 & 0.7 \\
\hline $\begin{array}{l}\text { Relación horizontal } \\
\text { multilateral }\end{array}$ & 0.9 & 0.5 & 0.0 & 1.8 & 0.8 \\
\hline $\begin{array}{l}\text { Índice de acción } \\
\text { conjunta }\end{array}$ & 5.4 & 5.0 & 3.3 & 6.7 & 5.3 \\
\hline $\begin{array}{l}\text { Índice de eficiencia } \\
\text { colectiva }^{1}\end{array}$ & 5.8 & 5.5 & 4.7 & 7.4 & 5.8 \\
\hline
\end{tabular}

Nota: ${ }^{1 /}$ Índice de eficiencia colectiva $=0.5$ índice de economías externas +0.5 índice acción conjunta. Fuente: Elaboración propia con base en datos obtenidos en el trabajo de campo.

La capacidad de innovar se analiza como factor de generación de conocimiento productivo, lo que coadyuva al desarrollo de empresas competitivas sustentables (Villarreal, 2003). Se observa que en el incipiente cluster del tomate bajo agricultura protegida se podría generar en el mediano plazo un grupo de trabajadores especializados que beneficie tanto a los trabajadores como a las agroempresas, como ha sido el caso del cluster de frutas en el Brasil (Gomes, 2006). Lo que está siendo apoyado por las instituciones de educación en el estado a través de la oferta de una especialización, diplomados y cursos especializados en el área de la agricultura protegida; sin embargo, actualmente se reconoce una alta desvinculación con los centros de investigación locales. Debe señalarse que, aunque existe buena 
disposición de los centros de investigación y desarrollo tecnológico en el estado, existe poca comunicación entre éstos y las unidades de producción. Con base en el trabajo de campo, sólo el $22 \%$ de éstas tiene vinculación con algún centro de investigación o universidad, la mayor parte de las agroempresas reciben el apoyo tecnológico de los proveedores, los cuales muchos son extranjeros. También se encontró que el $82 \%$ de las unidades de producción fueron construidas por proveedores extranjeros, en su gran mayoría empresas constructoras españolas. De igual forma, el $62 \%$ de la tecnología de producción y el 58\% del origen de la semilla son de proveedores españoles, estadounidenses, israelitas y canadienses principalmente, lo que muestra gran dependencia tecnológica del exterior. En lo que se refiere al mercado, las empresas que conocen y cumplen con los estándares y regulaciones establecidos en los mercados internacionales tienen un mejor acceso tanto en el mercado local como en los de exportación.

Las economías externas no explican por sí solas a los clusters exitosos, sino que es necesario considerar la acción conjunta y deliberada de los agentes. Esta cooperación implica un desarrollo gradual de confianza, la que forma parte de un proceso integral en donde las empresas desarrollan relaciones cooperativas de largo plazo y establecen principios para guiar su respuesta ante la incertidumbre (Villarreal, 2007). La acción conjunta en el cluster de tomate bajo agricultura protegida es muy débil; se manifiestan pocas acciones de coordinación voluntaria entre las unidades de producción y demás agentes con los que se relacionan. El IAC se encuentra por debajo de lo reportado por los clusters de frutas brasileños (Pietrobelli et al., 2006). Debido a la buena relación y apoyo que los productores reciben tanto de los clientes como de los proveedores - financiamiento y tecnológico- el eslabonamiento hacia adelante y hacia atrás son las principales fuentes de acción conjunta; esto se hace más evidente en el grupo de las unidades de producción de alta tecnología. Los bajos valores registrados en el ámbito de la acción conjunta denotan la baja colaboración entre los productores y muestran la necesidad de clarificar y fortalecer el papel de las asociaciones de productores en el estado de Zacatecas, así como de fomentar su creación bajo un entendimiento del papel que éstas juegan para el desarrollo de la competitividad de la industria.

\section{Cadenas de valor y gobernanza}

El tipo de gobernanza o coordinación de mercado es el que prevalece en la cadena de valor de las unidades de producción bajo agricultura protegida. Considerando la agrupación por nivel tecnológico, para las unidades de producción de alta tec- 
nología insertadas en una cadena global de valor, al igual que en otros clusters basados en recursos naturales (Gomes, 2006), el tipo de gobernanza más común es el de cuasi-jerarquía con compradores internacionales como líderes (cuadro 3). Los compradores internacionales facilitan la relación con los mercados extranjeros señalando la necesidad y los modos del upgrading requerido (Pietrobelli et al., 2006); éstos toman en cuenta los estándares que se deben cumplir, pero normalmente no apoyan el proceso de upgrading de la empresa; más bien los compradores seleccionan la empresa que tenga las condiciones de cumplir con los estándares establecidos. En el cluster del tomate analizado la forma de gobernanza de redes también está presente, aunque con poca incidencia.

\section{Cuadro 3}

Tipos de gobernanza de la cadena de valor en las que participan las unidades de producción agrupadas por nivel tecnológico

\begin{tabular}{l|c|c|c|c|c}
\hline $\begin{array}{c}\text { Tipo de } \\
\text { gobernanza }\end{array}$ & $\begin{array}{c}\text { Baja } \\
\text { tecnología }\end{array}$ & $\begin{array}{c}\text { Tecnología } \\
\text { intermedia }\end{array}$ & $\begin{array}{c}\text { Transición } \\
\text { tecnológica }\end{array}$ & $\begin{array}{c}\text { Alta } \\
\text { tecnología }\end{array}$ & $\begin{array}{c}\text { Promedio } \\
\text { total }\end{array}$ \\
\hline Mercado & 0.9 & 1.0 & 1.0 & 0.7 & 0.9 \\
Redes & 0.2 & 0.3 & 0.7 & 0.3 & 0.3 \\
Cuasi-jerarquía & 0.5 & 0.0 & 0.0 & 1.8 & 0.5 \\
Jerarquía & 0.0 & 0.0 & 0.0 & 0.0 & 0.0 \\
\hline
\end{tabular}

Nota: Se presenta el promedio de los valores registrados en cada una de las unidades de producción incluidas en cada grupo, en donde $0=$ no existe, $1=$ cadena doméstica; $2=$ cadena global; $3=$ cadena doméstica y global. Algunas unidades de producción participan en más de una cadena de valor.

Fuente: Elaboración propia con base en datos obtenidos en el trabajo de campo.

\section{Upgrading y eficiencia colectiva}

En las unidades de producción estudiadas sólo se reportó upgrading de proceso o de producto, situación similar a la que encontró Pietrobelli et al. (2006) en los clusters brasileños de melón y de manzanas. No se presentaron casos del upgrading funcional o intersectorial. Como se esperaba, los valores más elevados se registraron en el grupo de unidades de producción de alta tecnología (cuadro 4).

El grupo de agroempresas de alta tecnología tienen un buen manejo del cultivo y una alta disposición por parte de sus directivos para cambiar o mejorarlo con base en los requerimientos de la demanda y las normas establecidas en el mercado; aunque también se reconoce la existencia de problemas en el proceso de produc- 
ción sobre todo en los primeros años de la adopción de una nueva tecnología. De acuerdo con Cook y Calvin (2005), los nuevos productores en el negocio de los invernaderos no logran de inmediato los rendimientos potenciales esperados en la adopción de tecnologías que aplican; en esta industria, la curva de aprendizaje es de tres a cinco años, aspecto que se espera abatir en el mediano plazo. En el caso de los otros grupos tecnológicos, éstos presentan menor capacidad de innovar y más reducido acceso a canales de comercialización; sin embargo, aunque leves, estas agroempresas registran acciones de mejoras tanto en el producto como en sus procesos. Esta situación sugiere mayor promoción de acciones que generen eficiencia colectiva que fortalezca el upgrading, tales como capacitación para mejorar los procesos de la comercialización del tomate, así como sobre la aplicación de las buenas prácticas agrícolas y de manufactura.

De acuerdo con Pietrobelli et al. (2006), la evidencia empírica sugiere que las diferentes industrias por sectores tienden a mostrar diferentes niveles de eficiencia colectiva y un rango de gobernanza; situación similar se observa cuando se realiza este análisis por grupo tecnológico. En el cluster de tomate bajo agricultura protegida, la eficiencia colectiva y el upgrading se encuentran positivamente correlacionados (cuadro 3); sin embargo, cuando se analiza por grupo tecnológico, contrario a lo esperado, la relación entre la eficiencia colectiva y el upgrading en los grupos de transición tecnológica y tecnología alta es no estadísticamente significativa (cuadro 4); esto se puede atribuir al comportamiento individual y cerrado de algunas empresas de este grupo y al nivel de acciones conjuntas y economías externas que reportan. En el caso de los grupos de baja tecnología y tecnología intermedia hay una relación positiva y estadísticamente significativa entre los conceptos en mención que se atribuye a que en estos grupos se encuentran varias unidades de producción grandes y dos de éstas son empresas líderes en el sector. Los propietarios son los promotores de la primera integradora de invernaderos en el estado y están experimentando con diferentes modalidades de estructuras y variedades del cultivo. Algunos de ellos aún no exportan, sin embargo, han manifestado su interés por lograrlo en el mediano plazo. Esto se ha traducido en algunas acciones conjuntas que impactan la eficiencia colectiva de estos grupos y se reflejan en el upgrading de procesos o producto. 


\section{Cuadro 4}

\section{Upgrading y eficiencia colectiva en el las unidades de producción} bajo agricultura protegida agrupadas por nivel tecnológico

\begin{tabular}{|c|c|c|c|c|c|c|}
\hline $\begin{array}{c}\text { Tipo de } \\
\text { upgrading }\end{array}$ & $\begin{array}{c}\text { Baja } \\
\text { tecnología }\end{array}$ & $\begin{array}{l}\text { Tecnología } \\
\text { intermedia }\end{array}$ & $\begin{array}{l}\text { Transición } \\
\text { tecnológica }\end{array}$ & $\begin{array}{c}\text { Alta } \\
\text { tecnología }\end{array}$ & $\begin{array}{c}\text { Promedio } \\
\text { total }\end{array}$ & $\begin{array}{l}\text { Coeficiente } \\
\text { correlación }\end{array}$ \\
\hline Proceso & 0.6 & 0.3 & 1.3 & 2.3 & 0.8 & $0.39 * *$ \\
\hline Producto & 1.5 & 1.5 & 1.0 & 2.3 & 1.6 & $0.58 * *$ \\
\hline Funcional & 0.0 & 0.0 & 0.0 & 0.0 & 0.0 & - \\
\hline Intersectorial & 0.0 & 0.0 & 0.0 & 0.0 & 0.0 & - \\
\hline Total & 2.1 & 1.8 & 2.3 & 4.7 & 2.3 & \\
\hline $\begin{array}{l}\text { Coeficiente de } \\
\text { correlación } \\
\text { upgrading y } \\
\text { eficiencia colectiva }\end{array}$ & $0.50 *$ & $0.90 * *$ & 0.50 & -0.53 & $0.60 * *$ & \\
\hline $\begin{array}{l}\text { Núm. unidades de } \\
\text { producción }\end{array}$ & 24 & 12 & 3 & 6 & 45 & \\
\hline
\end{tabular}

Nota: Se presenta el promedio de los valores registrados en cada una de las unidades de producción incluidas en cada grupo, en donde $0=$ no existe, $1=$ bajo; $2=$ medio; $3=$ alto.

*/ La correlación es significativa al 0.05 . **/ La correlación es significativa al 0.01.

Fuente: Elaboración propia con base en datos obtenidos en el trabajo de campo.

\section{Conclusiones}

Contar con alto nivel tecnológico en los sistemas de producción bajo agricultura protegida es una condición necesaria, más no suficiente, para que éstas permanezcan competitivas en el mercado global; para lograrlo, el upgrading de procesos y productos, aunado a la realización de acciones de economías externas y acciones conjuntas, son necesarias.

Al igual que en otros clusters basados en recursos naturales de América Latina, en el naciente cluster de tomate las economías externas son mucho más frecuentes que las acciones conjuntas, aunque los valores obtenidos se encuentran un $50 \%$ por debajo de los valores máximos esperados. Esto se puede atribuir a lo complejo y largo que es el proceso de construir confianza y desarrollar localmente capital social. En esta etapa inicial del cluster de tomate bajo agricultura protegida, las organizaciones públicas serían las mediadoras con las empresas para que participen en proyectos conjuntos. 
La eficiencia colectiva y el upgrading están relacionados; se debe fortalecer la capacidad de innovación, por tal motivo las innovaciones tecnológicas debieran difundirse a través de la vinculación con las universidades, centros de investigación y asociaciones de productores. De igual forma, se considera importante promover la confianza y colaboración entre productores, así como fomentar la creación de asociaciones considerando el papel de éstas en el desarrollo de una competitividad sostenible.

No obstante que los compradores internacionales son el vínculo de las unidades de producción hacia el mercado internacional, éstos sólo han señalado la necesidad y los modos del upgrading requerido debido a que las normas y estándares de los mercados internacionales ya han sido establecidos. Los compradores en el mercado nacional también sólo señalan las formas de upgrading, sin participar en inversión en las unidades de producción, ya que implicaría elevar sus costos de transacción.

Las acciones que se sugieren para el apoyo de políticas que coadyuven al fomento de la competitividad del cluster serían aquellas que faciliten el desarrollo de las economías externas, promuevan el encadenamiento entre las empresas y fortalezcan la posición local dentro de la cadena de valor. Las propuestas son las siguientes: a) definición de acciones específicas para los nuevos entrantes que les ayude a reducir la curva de aprendizaje dentro del sector (servicios de extensión, capacitación, establecimiento de unidad de producción escuela coordinada por algún centro de investigación estatal, entre otras); b) crear y fortalecer la confianza entre las empresas y promover el establecimiento de proyectos colectivos; en este caso, la asistencia técnica especializada es extremadamente valuable y debe ser promovida por agentes o instituciones con un alta habilidad técnica y que goce de credibilidad con los productores locales; c) crear y fortalecer asociaciones de productores; las asociaciones de productores son la voz colectiva del cluster que fortalecen la acción conjunta; d) mayor difusión de los avances científicos del área, de las normas y estándares requeridos al tomate por los compradores nacionales e internacionales, así como de las buenas prácticas agrícolas y de manufactura, lo que implica mayor vinculación con instituciones de educación superior y centros de investigación; y e) inversión pública y privada en el desarrollo tecnológico en el sector. 


\section{Referencias}

Brambila Paz, J. J. (2006). En el umbral de una agricultura nueva. Estado de México: UACH, Colegio de Posgraduados.

Calvin, L. y R. Cook (coord.). (2001). U.S. fresh fruit and vegetable marketing: emerging trade practices, trends, and issues. Economic Research Service, USDA. January, Agricultural Economic Report (795).

Coelho, A. (2007). Eficiencia colectiva y upgrading en el cluster de tequila. Revista Análisis Económico XXII (049): 169-194.

Cook, R. y L. Calvin (2005). Greenhouse tomatoes change the dynamics of the North American fresh tomato industry. Economic Research Report, USDA (2). [En línea] http_//ww.ers.usda.gov/publications/err2/. Consultado en agosto de 2005 .

Cooke, P. y R. Huggins (2002). High technology clustering in Cambridge (UK). S.A. Amin, F. Goglio y A. Sforzi (Eds.). The institutions of local development (IGU series on local development). England: Ashgate Publishing Limited.

Gobierno del Estado de Zacatecas (1999). Plan Estatal de Desarrollo 1999- 2004. Zacatecas: GODEZAC.

(2005). Plan Estatal de Desarrollo 2005- 2010. Zacatecas: GODEZAC.

Gomes, R. (2006). Upgrading without exclusion: Lessons from SMEs in fresh fruit producing clusters in Brazil. C. Pietrobelli y R. Rabelloti (Eds.). Upgrading to compete. Global value chains, clusters, and SMEs in Latin America. Washingthon. D.C.: Inter-American Development Bank. David Rockefeller Center for Latin American Studies Harvard University.

Giuliani, E., C. Pietrobelli y R. Rabellotti (2005). Upgrading in global value chains: lessons from Latin American clusters. World Development 33 (4): 549-573. 
Humphrey, J. y H. Schmitz (2000). Governance and upgrading: Linking industrial cluster and global value chain research. IDS Working Paper, 120. Brighton: Institute of Development Studies, University of Sussex.

(2002). Developing country firms in the world economy: Governance and upgrading in global value chains. INEF Report (61). Duisburg: University of Duisburg. [En línea] http://www.ids.ac.uk/ids/global/vwpap.html. Consultado julio de 2007.

Kaufman, P., Ch. Handy, E. McLaughlin, K. Park y G. Green (2000). Understanding the dynamic of produce markets: Consumption and consolidation grow. Agosto, AEB (758). ERS-USDA.

Kinsey, J. (2005). Tendencias en la economía de alimentos. Comercio Exterior 55 (3): 249-253.

Nadvi, K. (1999). The cutting edge: Collective efficiency and international competitiveness in Pakistan. Oxford Development Studies 27: 81-107.

Padilla-Bernal, L.E., A.F. Rumayor-Rodríguez, O. Pérez-Veyna y E. Reyes-Rivas (2008). La competitividad de la industria del tomate bajo agricultura protegida de Zacatecas. Informe Técnico. Zacatecas: Fundación Produce Zacatecas.

Pietrobelli, C. y R. Rabellotti (2004). Upgrading in clusters and value chains in Latin America: the role of policies. Inter-American Development Bank: Washington, D.C. [En línea] http://cec.uniandes.edu.co/docs/ Upgrading\%20clusters.pdf. Consultado julio de 2007.

of an integrated approach. En C. Pietrobelli y R. Rabelloti (Eds.). Upgrading to compete. Global value chains, clusters, and SMEs in Latin América. Washingthon, D.C.: Inter-American Development Bank. David Rockefeller Center for Latin American Studies Harvard University. 
y E. Giulliani (2006). Upgrading in global value chains. Lessons from Latin American Clusters. C. Pietrobelli y R. Rabelloti (eds.). Upgrading to compete. Global value chains, clusters, and SMEs in Latin América. Washingthon, D.C.: Inter-American Development Bank. David Rockefeller Center for Latin American Studies Harvard University.

Schmitz, H. (1995). Collective efficiency: Growth path for small-scale industry. Journal of Development Studies 31: 529-566.

Suárez, R. y E. Bejarano (2001). Modelos de organización de empresas agropecuarias. CEGA, Documentos de trabajo (9): 184pp. [En línea] http://ww.cega. org.co/investigaciones_y_estudios/pdf/doc9.pdf. Consultado diciembre de 2005

Villarreal, R. y T. Villarreal (2003). IFA: La empresa competitiva sustentable en la era del capital intelectual. México: McGraw Hill Interamericana.

Villarreal, R. (2007). El paradigma de la competitividad sistémica. En J.L. Calva (coord.). Educación, ciencia, tecnología y competitividad. México: UNAM, Cámara de Diputados, LX Legislatura.

Visser, E.J. (2004). A chilean wine cluster? Governance and upgrading in the phase of internationalization. Santiago de Chile: ECLAC. United Nations Publications. 
